
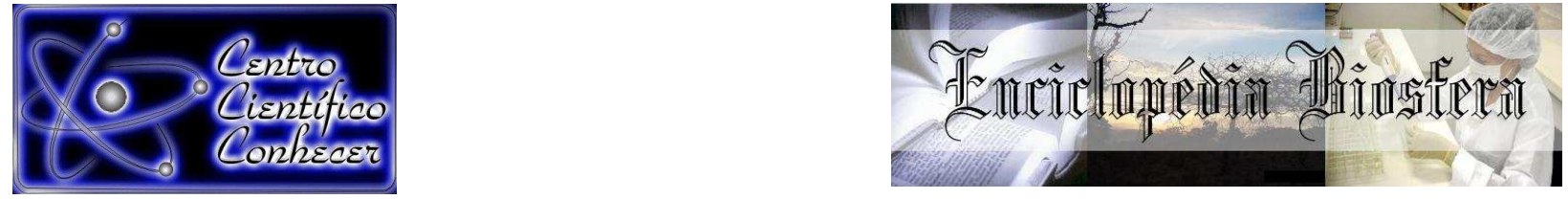

\title{
DETERMINAÇÃO DOS ATRIBUTOS QUÍMICOS DO SOLO DE ILHAS DO LAGO DA USINA HIDRELÉTRICA DE TUCURUÍ-PA
}

\author{
Camila Lorrane Gaia Veras ${ }^{1}$, Sandra Andréa Santos da Silva², Yarnel de Oliveira \\ Campos $^{3}$
}

${ }^{1}$ Graduanda do curso de Engenharia Sanitária e Ambiental, na Universidade Federal do Pará - UFPA, Campus de Tucuruí. Email: camilalorraneveras@gmail.com 2Profa Doutora da Universidade Federal do Pará - UFPA, Campus de Altamira. 3Prof. Doutor da Universidade Federal do Pará - UFPA, Campus de Tucuruí.

Recebido em: 08/04/2016 - Aprovado em: 30/05/2016 - Publicado em: 20/06/2016 DOI: 10.18677/Enciclopedia_Biosfera_2016_041

\begin{abstract}
A construção da Usina Hidrelétrica de Tucuruí-PA e seu reservatório resultaram na criação de ilhas artificiais que ao longo do tempo tem sofrido mudanças com o uso e ocupação da área por comunidades ribeirinhas e o aumento do nível de água durante os períodos de chuva. Esses processos resultam em alterações que acarretam na perda da capacidade produtiva do solo, como a erosão hídrica, acidificação dos solos e exaustão de nutrientes, tornando-o inviável para a exploração socioeconômica e ambiental. Os estudos e análises acerca das características do solo fornecem informações que contribuem para o uso racional e conservação de ecossistemas sustentáveis. Objetivou-se com esse trabalho realizar uma análise sobre as alterações nos ecossistemas naturais, utilizando indicadores químicos e fertilidade no solo das ilhas do lago da UHT de Tucuruí -PA,. Foram coletadas amostras entre $0-40 \mathrm{~cm}$, em um transecto que seguia desde à margem da ilha até o ponto de vegetação, e analisados os aspectos químicos que contribuem na estrutura e fertilidade do solo. A análise resultou em um solo degradado pelo processo de lixiviação, com níveis muito baixos de $\mathrm{P}$ e $\mathrm{C}$, devido aos processos de inundação que ocorrem nos períodos de chuva e aumento do nível do lago. A escassez de nutrientes nas áreas de vegetação, e a influência da saturação hídrica na qualidade do solo às margens do lago sugerem um solo com pouca estrutura e resistência contra fenômenos de erosão e assoreamento.
\end{abstract}

PALAVRAS-CHAVE: Erosão Hídrica, fertilidade do solo, solos de ilhas. 


\title{
SOIL CHEMICAL ATTRIBUTES IN ISLANDS FROM A HYDROELETRICAL POWER PLANT LAKE, IN TUCURUÍ-PA
}

\begin{abstract}
The construction of the hydroelectric plant in Tucuruí, Brazil, and its artificial lake resulted in the creation of islands that over time has been changed by the area's use and occupation by the riverine communities and the increase of the water's level during the rainy season. These processes results in changes that cause the loss of the soil's productive ability, such as water erosion, soil acidification and the depletion of nutrients, making it unaffordable for socioeconomic and environmental exploitation. Studies and analyzes of soil characteristics provide information that may contributes to the rational use and conservation of sustainable ecosystems. This research aims to make a literature review about the changes on natural ecosystems using the chemical aspects and fertility in the soil at the islands of Tucurui's hydroelectric power plant, in Brazil. The samples were collected between a depth of $0-40 \mathrm{~cm}$, in a transect from the lakeshore straight to the vegetation area, and their chemical aspects that contribute to the structure and fertility of the soil were analyzed in the laboratory. The results revealed a degraded soil by leaching process, with very low levels of $P$ and organic $C$, due to the flooding that occurs during the rainy season and the increase of the lake level. The low levels of nutrients in the vegetation areas and the influence of water saturation in soil quality on the lakeshores suggest a soil with a poor structure and low strength to situations like erosion and siltation.
\end{abstract}

KEYWORDS: Island Soil, Soil Fertility, Hydro Erosion

\section{INTRODUÇÃO}

A ocupação das ilhas no entorno do lago da UHE Tucuruí, no estado do Pará aconteceu por volta do ano de 1986, após o enchimento do reservatório da usina. Foram formadas cerca de 1660 ilhas, quase o triplo do número previsto (JATOBÁ, 2006), que ao longo dos anos, foram habitadas por famílias que inicialmente viviam da pesca, e logo estabeleceram uma cultura de lavoura, caça e plantio. Em 2002, estabeleceu-se a criação de uma Área de Proteção Ambiental (APA) no conjunto de ilhas do lago da UHE, de forma a conciliar a ocupação e atividade humana com a sustentabilidade necessária neste ecossistema (ARAÚJO, 2008).

Os Latossolos se caracterizam por possuir grande permeabilidade e serem intemperizados, com pequena reserva de nutrientes para as plantas (CAETANO e al., 2013). Somando-se a pouca presença de vegetação ciliar e constante saturação de água, este tipo de solo se torna ainda mais passivo aos processos de lixiviação e erosão hídrica. Dentre os fatores causadores da erosão hídrica e lixiviamento está a perda de matéria orgânica do solo, fenômeno estritamente ligado à presença de cobertura ou de resíduos culturais remanescentes na superfície.

Segundo LEITE et al. (2009) a presença de cobertura vegetal contribui na estrutura dos agregados do solo, na redução do escoamento superficial e capacidade erosiva da enxurrada. $\mathrm{O}$ autor ainda ressalta que práticas constantes de cultivo e preparo do solo são os responsáveis pela diminuição significativa de nutrientes como nitrogênio, potássio e fósforo e carbono orgânico, contribuindo com a quebra dos agregados e exposição do solo, causando degradação de sua estrutura.

Os atributos químicos presentes nos solos de ilhas servem como base para indicar impactos causados pela ocupação e atividade humana, bem como o ENCICLOPÉDIA BIOSFERA, Centro Científico Conhecer - Goiânia, v.13 n.23; p. 459 2016 
desenvolvimento de processos naturais de intemperização, além de auxiliar no monitoramento de áreas que devem, por lei, serem preservadas.

Os indicadores de qualidade do solo são propriedades mensuráveis (quantitativas ou qualitativas) do solo ou da planta acerca de um processo ou atividade e que permitem caracterizar, avaliar e acompanhar as alterações ocorridas num dado ecossistema (KARLEN et al. 1997). Este trabalho teve como objetivo analisar as características químicas do solo das ilhas da região do lago da UHE Tucuruí, de modo a relacionar os dados obtidos com os processos de intemperização e mudanças naturais e antrópicas desenvolvidas nesta área ao longo do tempo. Dentre as propriedades constituintes de um determinado solo, temos a presença da ciclagem de nutrientes, matéria em decomposição, capacidade de troca catiônica, $\mathrm{pH}$ e saturação por base, que caracterizam a fertilidade, produção, e nutrição do solo e das plantas que nele vivem, bem como são constituintes dos indicadores de qualidade do solo.

\section{MATERIAL E METODOS}

A região das Ilhas da Usina Hidrelétrica do município de Tucuruí (UHE Tucuruí) localiza-se no sudeste do estado do Pará, com uma área territorial de aproximadamente $2.086 \mathrm{~km}^{2}$ (IBGE, 2014). A hidrografia do município baseia-se no lago artificial originado na barragem do Rio Tocantins, que consiste em $2.430 \mathrm{~km}^{2}$ contendo cerca de 45,8 bilhões de $\mathrm{m}^{3}$ de água. O clima se categoriza como equatorial super-úmido, tipo Ami, de acordo com a classificação de Köppen, com temperatura média anual de $26^{\circ} \mathrm{C}$, média máxima em torno de $32,0^{\circ} \mathrm{C}$ e mínima de 22,7ํㅡ (SEPOF, 2011).

A coleta das amostras de solo ocorreu em 12 de janeiro de 2015, durante 0 período de cheia do lago, em três ilhas denominadas P1, P2 e P3, apresentando a seguinte classificação: P1 - PV1: Podzólico Vermelho-Amarelo, P2 - PV3: Podzólico Vermelho-Amarelo e P3 - LV1: Latossolo Vermelho Amarelo, segundo o Plano Diretor de Tucuruí (2011). Em cada ilha realizou-se um transecto, com três pontos de coleta ( $A, B$ e $C$ ), sendo $A$ parte da ilha com maior altitude, $B$ uma altitude intermediária, e C localizada na borda da ilha, próximo à beira do lago (croqui abaixo). Foram retiradas duas amostras por ponto, classificadas pelas profundidades de $0-20 \mathrm{~cm}$ e $20-40 \mathrm{~cm}$. 


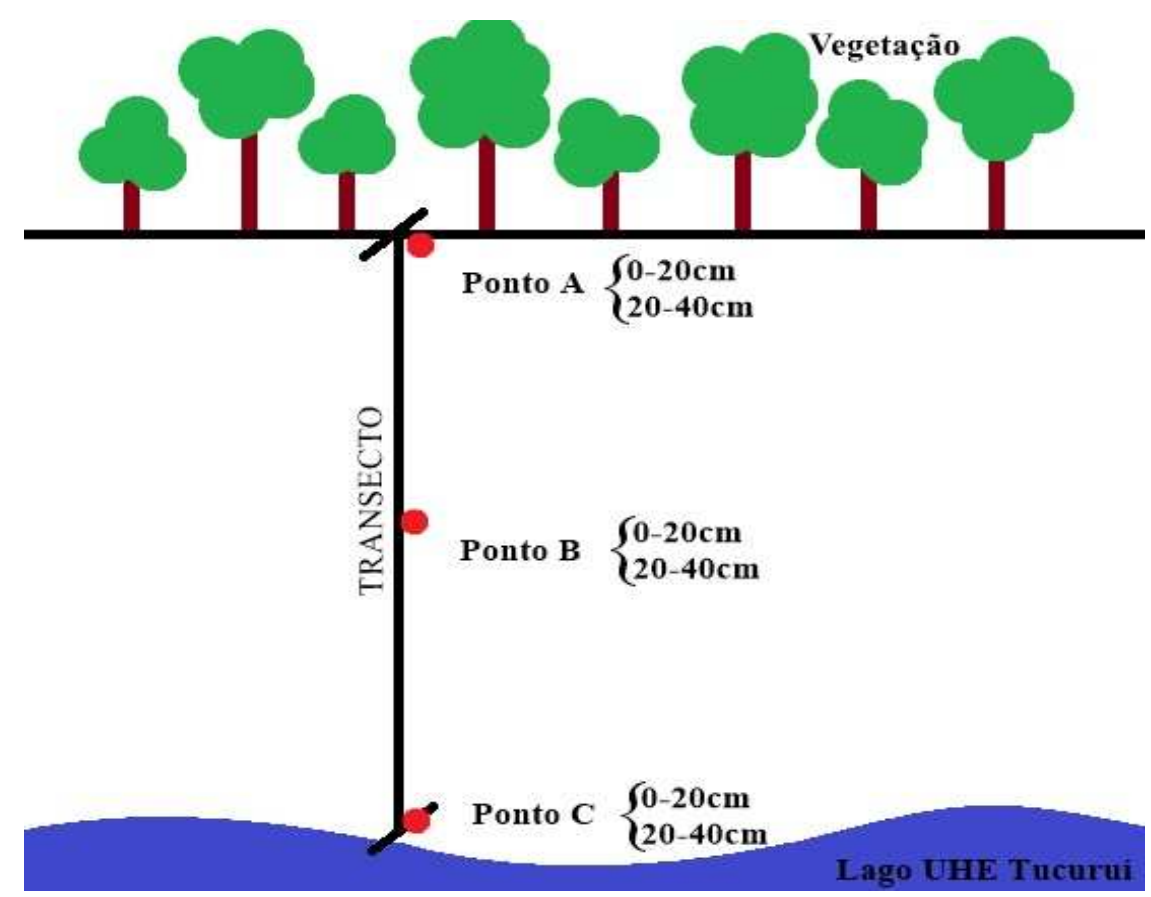

FIGURA 1: Croqui demonstrando a execução do transecto para a coleta das amostras.

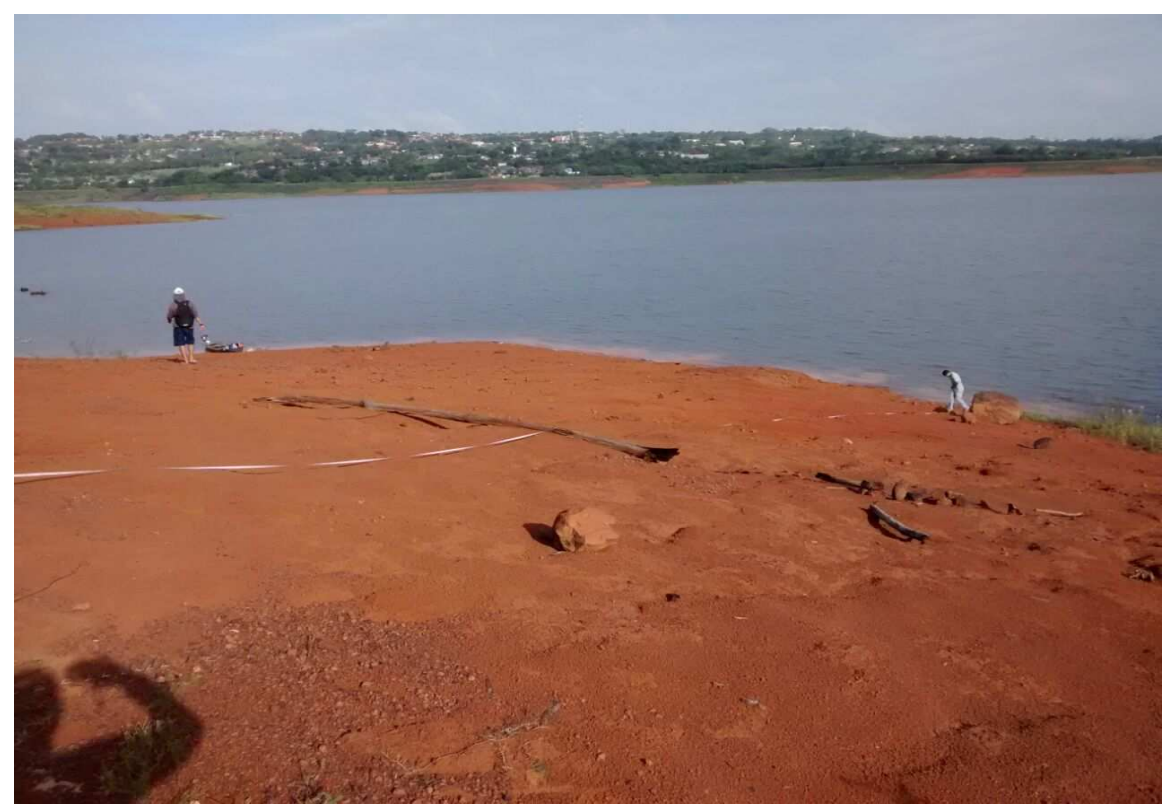

FIGURA 2: Método de coleta de amostras via transecto realizada em uma das áreas de estudo. 


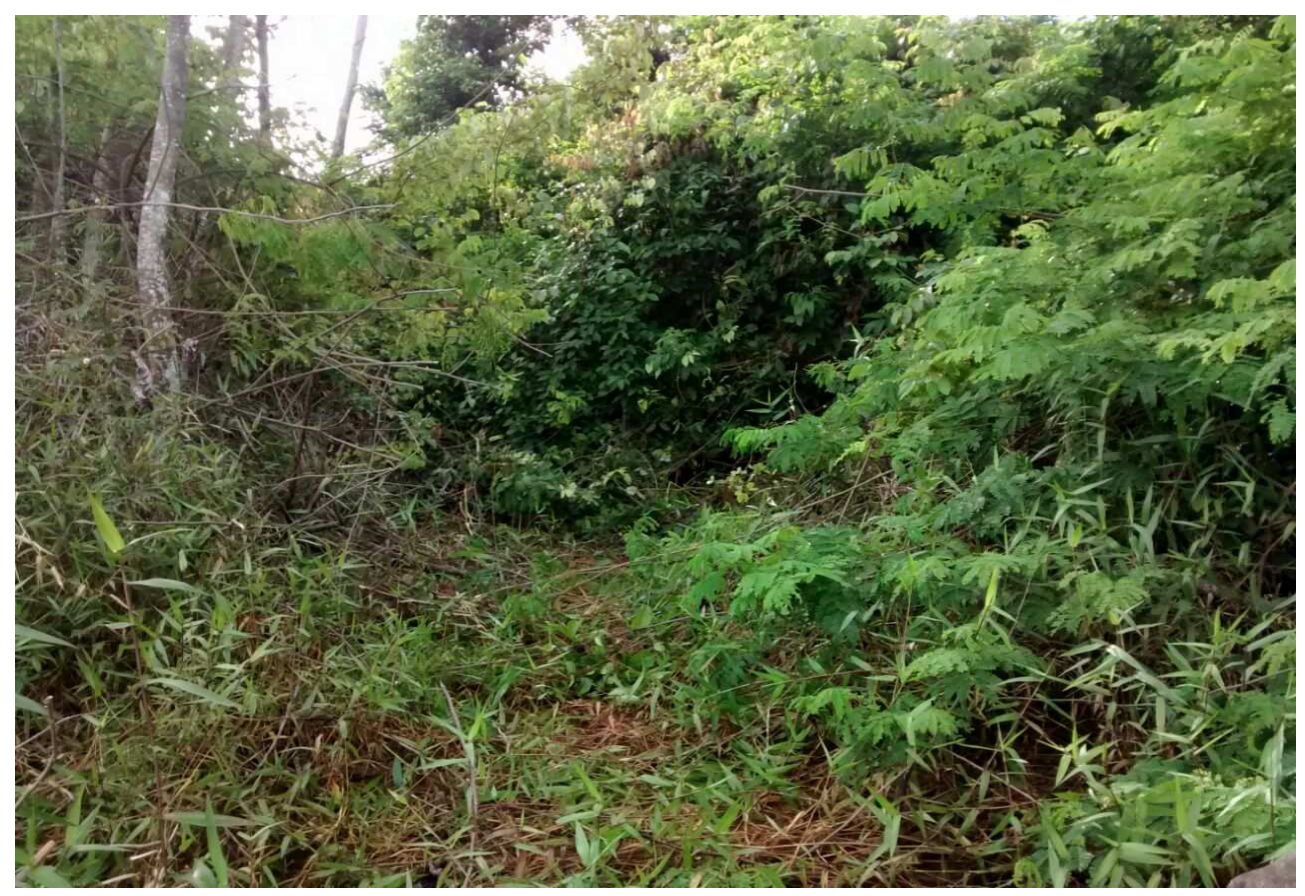

FIGURA 3: Vegetação existente nas áreas de coleta de solo.

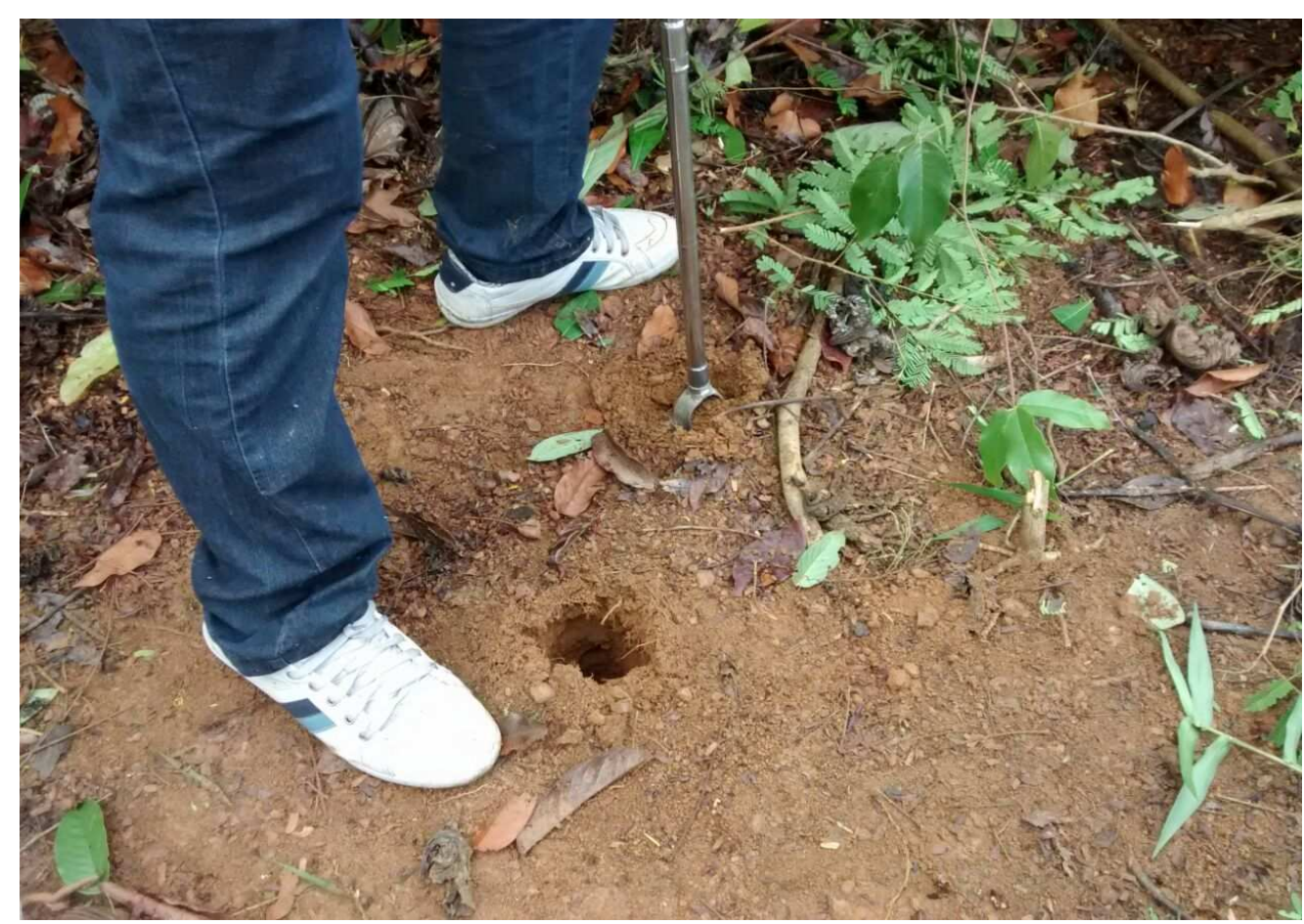

FIGURA 4: Coleta das amostras de solo.

Após coletadas, as amostras foram enviadas ao Laboratório de Solos do Instituto de Ciências Agrárias da Universidade Federal Rural da Amazônia, para a quantificação dos atributos químicos do solo, com a metodologia proposta pela EMBRAPA (2011). As análises estatísticas foram realizadas por meio do programa estatístico Minitab 17. Os atributos químicos abordados nesta análise foram: $\mathrm{pH}$, carbono orgânico, fósforo, potássio, cálcio, magnésio, alumínio, acidez potencial, 
soma de bases, capacidade de troca catiônica, saturação por bases e toxidez por alumínio

\section{RESULTADOS E DISCUSSÃO}

Os resultados estão descritos nas tabelas 1 e 2 , separadas pelas diferentes profundidades dos pontos de coleta.

TABELA 1: Teores dos atributos químicos do solo nas ilhas da UHE de Tucuruí PA, nas profundidades de 0-20 e 0-40 cm, no ano de 2015.

\begin{tabular}{|c|c|c|c|c|c|c|c|c|c|c|c|c|c|}
\hline \multirow[t]{2}{*}{ ID } & \multirow{2}{*}{$\begin{array}{l}\text { Prof. } \\
\text { (cm) }\end{array}$} & \multirow{2}{*}{$\begin{array}{l}\mathrm{pH} \\
\mathrm{H}_{2} \mathrm{O}\end{array}$} & \multirow{2}{*}{$\begin{array}{c}C_{\text {org }} \\
\text { g.kg- }{ }^{1}\end{array}$} & \multirow{2}{*}{$\begin{array}{c}P \\
\mathrm{mg} \cdot \mathrm{dm}^{-3}\end{array}$} & $\mathrm{~K}$ & $\mathrm{Ca}$ & $\mathbf{M g}$ & Al & $\mathrm{H}+\mathrm{Al}$ & SB & $\mathbf{T}$ & V & m \\
\hline & & & & & \multicolumn{7}{|c|}{ cmolc. $\mathrm{dm}^{-3}$} & \multicolumn{2}{|c|}{$\%$} \\
\hline \multirow{2}{*}{ P1A } & $0-20$ & 4,81 & 12,23 & 2,65 & 0,04 & 0,86 & 0,38 & 1,08 & 6,69 & 1,28 & 7,97 & 16,06 & 13,55 \\
\hline & $0-40$ & 4,81 & 9,78 & 1,99 & 0,02 & 0,67 & 0,19 & 0,96 & 6,16 & 0,88 & 7,04 & 12,50 & 13,63 \\
\hline \multirow{2}{*}{$\mathrm{P} 2 \mathrm{~A}$} & $0-20$ & 4,24 & 10,13 & 1,89 & 0,06 & 0,38 & 0,29 & 3,31 & 9,64 & 0,73 & 10,37 & 7,04 & 31,92 \\
\hline & $0-40$ & 4,21 & 12,58 & 1,43 & 0,04 & 0,29 & 0,29 & 2,95 & 8,57 & 0,62 & 9,19 & 6,74 & 32,10 \\
\hline \multirow{2}{*}{ P3A } & $0-20$ & 4,02 & 15,72 & 1,79 & 0,04 & 0,29 & 0,10 & 2,41 & 8,7 & 0,43 & 9,13 & 4,71 & 26,40 \\
\hline & $0-40$ & 4,16 & 10,83 & 0,87 & 0,03 & 0,29 & 0,10 & 2,11 & 6,43 & 0,42 & 6,85 & 6,13 & 30,80 \\
\hline \multirow{2}{*}{ P1B } & $0-20$ & 5,32 & 4,54 & 3,91 & 0,00 & 1,05 & 0,48 & 0,18 & 3,75 & 1,53 & 5,28 & 28,97 & 3,41 \\
\hline & $0-40$ & 5,60 & 4,54 & 5,58 & 0,01 & 1,24 & 0,67 & 0,00 & 2,54 & 1,92 & 4,46 & 43,04 & 0,00 \\
\hline \multirow{2}{*}{ P2B } & $0-20$ & 5,43 & 4,54 & 1,43 & 0,04 & 1,81 & 0,57 & 0,30 & 2,41 & 2,42 & 4,83 & 50,10 & 6,21 \\
\hline & $0-40$ & 5,34 & 2,10 & 1,38 & 0,02 & 1,24 & 0,57 & 0,12 & 3,08 & 1,83 & 4,91 & 37,27 & 2,44 \\
\hline \multirow{2}{*}{ P3B } & $0-20$ & 4,90 & 4,54 & 0,62 & 0,02 & 0,48 & 0,38 & 0,06 & 2,68 & 0,88 & 3,56 & 24,72 & 1,68 \\
\hline & $0-40$ & 4,94 & 5,59 & 0,62 & 0,00 & 0,48 & 0,38 & 0,66 & 3,21 & 0,86 & 4,07 & 21,13 & 16,21 \\
\hline \multirow{2}{*}{ P1C } & $0-20$ & 5,56 & 1,40 & 7,10 & 0,05 & 1,14 & 0,67 & 0,00 & 2,95 & 1,86 & 4,81 & 38,66 & 0,00 \\
\hline & $0-40$ & 5,51 & 1,75 & 7,26 & 0,05 & 1,05 & 0,67 & 0,00 & 3,35 & 1,77 & 5,12 & 34,57 & 0,00 \\
\hline \multirow{2}{*}{ P2C } & $0-20$ & 5,83 & 4,54 & 1,23 & 0,06 & 1,81 & 0,57 & 0,00 & 1,74 & 2,44 & 4,18 & 58,37 & 0,00 \\
\hline & $0-40$ & 6,03 & 1,75 & 2,09 & 0,03 & 2,00 & 0,76 & 0,00 & 1,34 & 2,79 & 4,13 & 67,55 & 0,00 \\
\hline \multirow{2}{*}{ P3C } & $0-20$ & 5,56 & 2,80 & 0,97 & 0,03 & 0,95 & 0,48 & 0,00 & 1,87 & 1,46 & 3,33 & 43,84 & 0,00 \\
\hline & $0-40$ & 5,46 & 2,45 & 0,77 & 0,02 & 0,29 & 0,10 & 0,06 & 2,41 & 0,41 & 2,82 & 14,54 & 2,12 \\
\hline
\end{tabular}

P1(A, B, C): solo tipo PV1, PV a A mod/arg pedreg I fond + LVA a A mod marg casc/marg ond + SP a A mod med/arg pedreg I fond (PT a A mod med/arg sond); P2(A, B, C): solo tipo PV3, PV a cn A mod med e arg casc pedreg lond + SP a A mod arg mt casc pedreg I ond (PT a A mod med/arg ond); P3(A, B, C): solo tipo LV1, LV a A mod marg pl + LV a cn A mod marg casc ond e sond + PV a A mod arg/marg ond + (SP a A mod med e arg mt casc ond); (Dados: Plano Diretor do Município de Tucuruí, 2015) ID: identificação das amostras de solo recolhidas; Prof.: profundidade da amostra no solo; H+Al: acidez potencial; SB: soma de bases trocáveis; T: capacidade de troca catiônica (CTC); $\mathbf{m}$ : toxidez por alumínio.

O valor de $\mathbf{p H}$ dos Latossolos e solos tropicais é naturalmente ácido, variando entre 4,0 e 5,5, sendo a correção da acidez essencial para o uso deste tipo de solo para fins agrícolas e preservação (EMBRAPA, 2015). O pH de Latossolos e solos tropicais, segundo a EMBRAPA (2015), varia entre 4,0 e 5,5. Nos pontos localizados à margem do lago o solo se apresentou mais alcalino, podendo estar relacionado à saturação hídrica, que favorece o aumento de pH. PAULA (2013) em seu estudo sobre florestas periodicamente inundadas obteve $\mathrm{pH}$ entre 3,89 e 4,42 valor inferior 
ao desta pesquisa. A maior acidez deste solo ocorre devido à quantidade superior de material de serapilheira acumulada na superfície, consequente dos alagamentos.

As amostras apresentaram pouca presença de $C$ orgânico, devido à falta de húmus, enquanto os pontos mais distantes da margem do lago (P2A e P3A) apresentaram valores mais elevados, devido à maior disponibilidade de vegetação, e consequentemente, de nutrientes. MATOSO et al. (2012), encontraram uma média de $13,34 \mathrm{~g} \cdot \mathrm{kg}^{-1}$ em áreas de vegetação natural, dados estes semelhantes aos desta pesquisa, que apresentou uma média de $11,87 \mathrm{~g} \cdot \mathrm{kg}^{-1}$ nos pontos em $A$, onde ocorre presença de vegetação nas diferentes ilhas. Outros fatores influentes na disponibilidade de C são a saturação hídrica e a atividade antrópica (NASCIMENTO et al., 2010), bem como o processo de arraste da camada superficial como consequência da erosão hídrica, fenômeno que se dá ao menos uma vez a cada semestre.

Os resultados apresentaram valores muito baixos de fósforo, tendo as amostras $\mathrm{P} 1(\mathrm{~A}, \mathrm{~B}, \mathrm{C})$ apresentado maiores teores devido à presença de matéria em decomposição, proveniente do material arrastado pelo processo de erosão hídrica a qual carrega conteúdo de serrapilheira de locais com presença de vegetação. COSTA (2015) relatou valores de fósforo entre 4 e $5 \mathrm{mg} \mathrm{dm}^{-3}$ em Latossolos de pastagem próximos à sub-bacias hidrográficas em processo de degradação ambiental, sendo interpretado como muito baixo, possui tendência a diminuir ainda mais, devido à redução das trocas de nutrientes causados pela ação antrópica. $O$ baixo valor de fósforo ressalta o estágio avançado de degradação do solo pelos processos erosivos nas ilhas estudadas. A ausência de vegetação somada à pouca quantidade de matéria orgânica e a acidez característica do solo podem ser a causa dos baixos teores de fósforo.

Os níveis de potássio não sofreram variações significativas entre as amostras nas ilhas estudadas, variando entre $0,00 \mathrm{cmol}_{\mathrm{c}} \cdot \mathrm{dm}^{-3}$ e $0,06 \mathrm{cmol}_{\mathrm{c}} \cdot \mathrm{dm}^{-3}$. Os baixos teores novamente apontaram a deficiência de nutrientes destes solos. SOUZA et al. (2015) obtiveram valores de potássio entre 0,06 e $0,23 \mathrm{cmol}_{\mathrm{c}} \cdot \mathrm{dm}^{-3}$, com os menores valores localizados na superfície dos pontos mais baixos das ilhas. As perdas de potássio no solo podem estar relacionadas ao fenômeno de lixiviação e a baixa CTC do solo (VILLA et al., 2004).

Segundo a EMBRAPA (2011), o teor de cálcio variou entre baixo e médio, com maiores valores nos pontos às margens do lago. COSTA et al. (2015) em Latossolos de pastagens sob degradação obtiveram valores entre 0,5 e 0,6 $\mathrm{cmol}_{\mathrm{c}} \cdot \mathrm{dm}^{-3}$, ressaltando que os valores de cálcio são diretamente influenciados pela quantidade de matéria orgânica presente no solo.

Por conseguinte, o magnésio variou entre teores baixos e médios (EMBRAPA, 2011), sendo encontrado em valores mais elevados nos pontos com maior $\mathrm{pH}$, onde há maior quantidade de matéria em decomposição e outros nutrientes. SOUSA et al. (2015) em pesquisa sobre erosão de solos de ilhas e margens de rios, obtiveram valores de magnésio entre 0,28 a $1,56 \mathrm{cmol}_{\mathrm{c}} \cdot \mathrm{dm}^{-3} \mathrm{em}$ neossolos fúlvicos, que apresentaram baixa fertilidade, proveniente apenas do material que é depositado e transportado pelos corpos hídricos.

$\mathrm{O}$ alúminio apresentou valores mais elevados, entre $0,96 \mathrm{cmol}_{\mathrm{c}} \cdot \mathrm{dm}^{-3}$ e 3,31 $\mathrm{cmol}_{\mathrm{c}} \cdot \mathrm{dm}^{-3}$, nos pontos mais próximos à vegetação, reduzindo em pontos mais próximo ao lago, com teores entre $0,00 \mathrm{cmol}_{\mathrm{c}} \cdot \mathrm{dm}^{-3}$ e $0,06 \mathrm{cmol}_{\mathrm{c}} \cdot \mathrm{dm}^{-3}$. BORGES et al. (2014) em solos de mata ciliar de ecossistema semelhante, obtiveram resultados variantes entre 0,2 e $1,65 \mathrm{cmol}_{\mathrm{c}} \cdot \mathrm{dm}^{-3}$, valores que indicam um nível de toxicidade às plantas. 
Os níveis de acidez potencial se apresentaram semelhantes aos do alumínio vistos anteriormente, com maior concentração nas amostras próxima às plantas, variando entre bom e muito bom, segundo os parâmetros da EMBRAPA (2011). Segundo BORGES et al. (2014) a acidez potencial em análise de um ecossistema semelhante apresentou cerca de $10,7 \mathrm{cmol}_{\mathrm{c}} \cdot \mathrm{dm}^{-3}$ na camada entre $0-20 \mathrm{~cm}$ e 6,05 $\mathrm{cmol}_{\mathrm{c}} \cdot \mathrm{dm}^{-3}$ na camada entre $20-40 \mathrm{~cm}$, valores superiores a este trabalho, que obteve uma média de $4,49 \mathrm{cmol}_{c} \cdot \mathrm{dm}^{-3}$ na profundidade entre $0-20 \mathrm{~cm}$ e 4,12 $\mathrm{cmol}_{\mathrm{c}} \cdot \mathrm{dm}^{-3}$ na profundidade entre $20-40 \mathrm{~cm}$.

A soma de bases se apresentou mais elevada nos pontos mais próximos ao lago, onde há maior saturação hídrica e um pH mais elevado. BORGES et al. (2014) obtiveram valores entre $8,15 \mathrm{cmol}_{\mathrm{c}} \cdot \mathrm{dm}^{-3}$ e $5,15 \mathrm{cmol}_{\mathrm{c}} \cdot \mathrm{dm}^{-3}$ em mata ciliar de ecossistema semelhante. Assim como SB, a saturação por base foi mais elevada nos pontos mais próximos do lago. Os valores elevados de V\% estão relacionados ao $\mathrm{pH}$ mais alcalino, bem como aos teores de $\mathrm{Ca}$ e $\mathrm{Mg}$ disponíveis no solo.

Houve uma maior concentração de capacidade de troca catiônica nos pontos próximos à vegetação, com maior CTC concentrada na superfície de $0-20 \mathrm{~cm}$, entre 6,69 $\mathrm{cmol}_{\mathrm{c}} \cdot \mathrm{dm}^{-3}$ e $9,64 \mathrm{cmol}_{\mathrm{c}} \cdot \mathrm{dm}^{-3}$, e na camada de $20-40 \mathrm{~cm}$ valores de CTC entre $6,16 \mathrm{cmol}_{\mathrm{c}} \cdot \mathrm{dm}^{-3}$ e $8,57 \mathrm{cmol}_{\mathrm{c}} \cdot \mathrm{dm}^{-3}$. No restante das amostras, obteve-se valores menores entre $1,34 \mathrm{cmol}_{\mathrm{c}} \cdot \mathrm{dm}^{-3}$ e $3,75 \mathrm{cmol}_{\mathrm{c}} \cdot \mathrm{dm}^{-3}$, com pouca variação entre as profundidades. Em solos de reflorestamento de vegetação ciliar, SILVA et al. (2012) relatou valores médios entre 6,3 e $8,0 \mathrm{cmol}_{\mathrm{c}} \cdot \mathrm{dm}^{-3}$.

Segundo a classificação da EMBRAPA (2009), os valores desta análise mostraram saturação por alumínio entre baixa e muito baixa na maioria das amostras coletadas, com exceção dos pontos P2A em 0-20 cm e 20-40 cm, e P3A em $0-40 \mathrm{~cm}$. Não houve variações significativas dos valores em relação à profundidade.

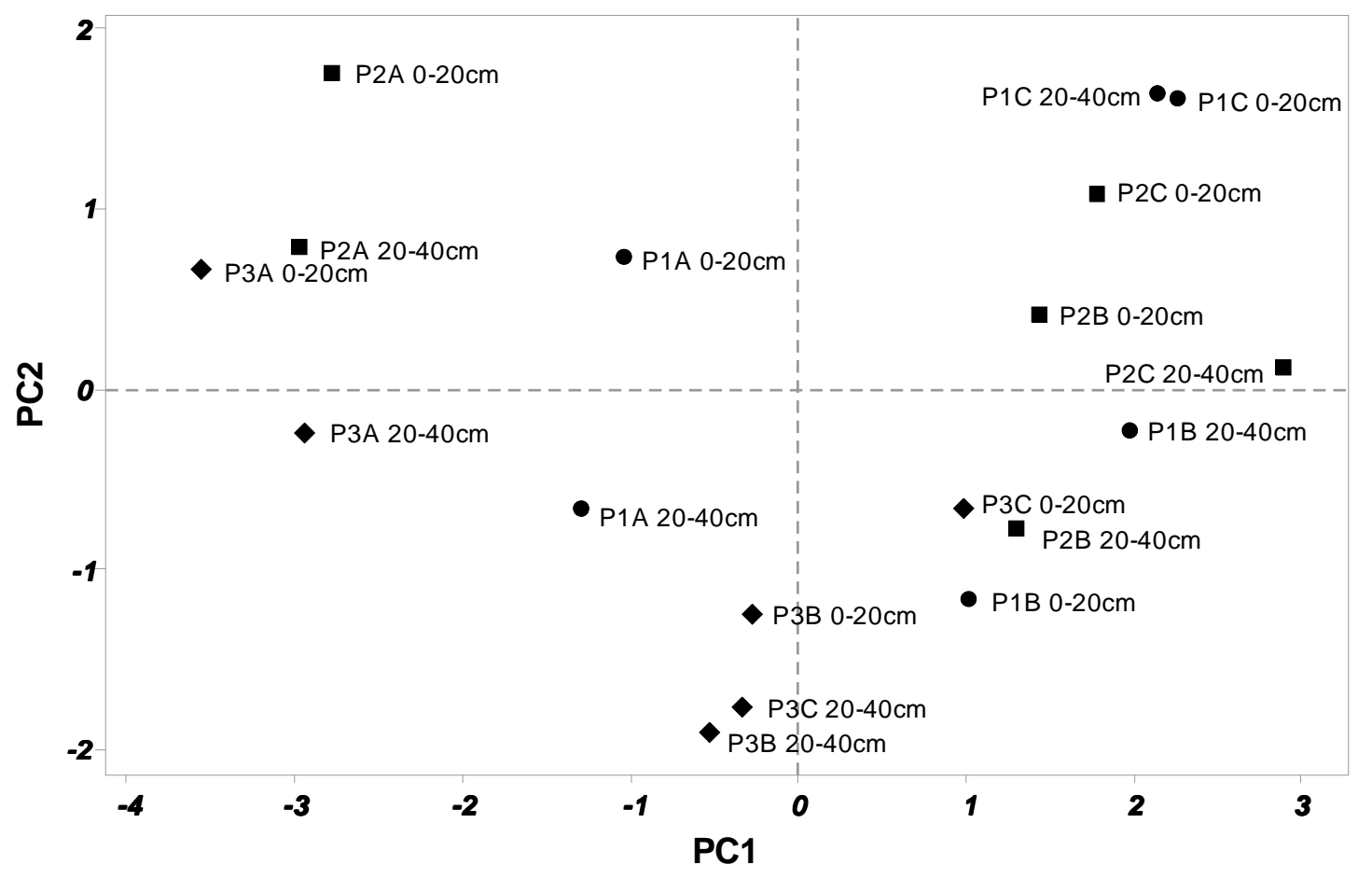

GRÁFICO 1: Principais componentes distribuídos em gráfico de scores, analisando os nutrientes $\mathrm{C}_{\text {org }}, \mathrm{P}, \mathrm{K}, \mathrm{Ca}, \mathrm{Mg}$ e Al. 


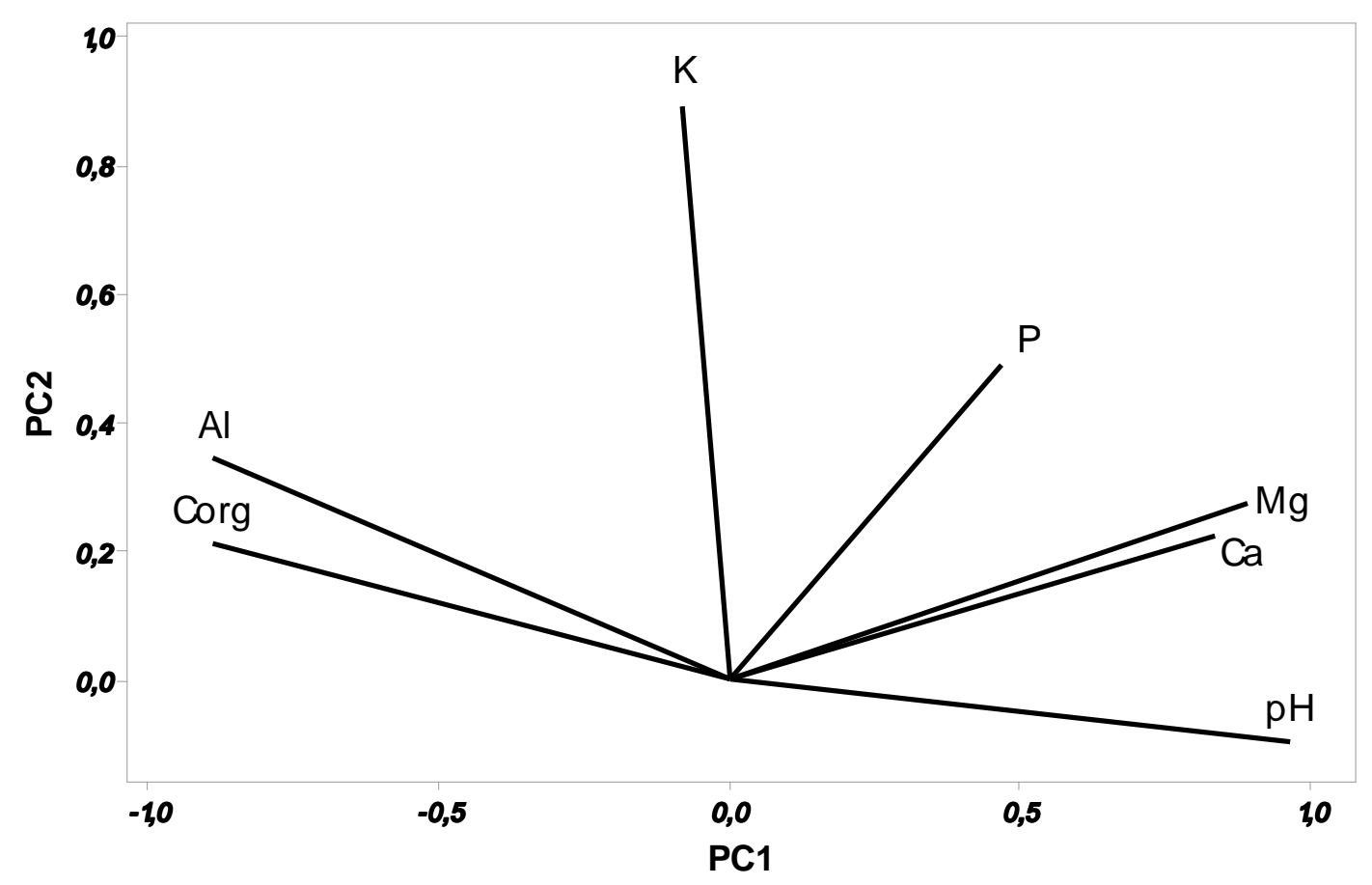

GRÁFICO 2: Gráfico dos pesos pra as duas primeiras componentes principais.

Analisando o gráfico dos escores (Gráfico 1) para as componentes principais das amostras (PC1 versus PC2) obtêm-se uma divisão entre as amostras que foram coletadas em pontos de alta topografia e as amostras que foram coletadas em pontos de topografia média (pontos em B) e baixa (pontos em C). O valor de PC1 onde ocorre essa divisão é próximo a -1.

As equações de PC1 (Eq.1) e de PC2 (Eq. 2) trazem os valores dos pesos para essas duas componentes.

$$
\begin{aligned}
& \text { PC } 1(60.4 \%)=0.47 \mathrm{pH}_{\mathrm{H} 2 \mathrm{O}}-0.43 \mathrm{C}+0.23 \mathrm{P}-0.04 \mathrm{~K}+0.41 \mathrm{Ca}+0.44 \mathrm{Mg}-0.43 \mathrm{Al} \\
& \mathrm{PC} 2(19.1 \%)=-0.09 \mathrm{pH}_{\mathrm{H} 2 \mathrm{O}}+0.18 \mathrm{C}+0.42 \mathrm{P}+0.77 \mathrm{~K}+0.19 \mathrm{Ca}+0.24 \mathrm{Mg}+0.30 \mathrm{Al}
\end{aligned}
$$

As variáveis que mais contribuem para a separação em $\mathrm{PC} 1$ são o $\mathrm{pH}, \mathrm{Ca}$, $\mathrm{Mg}, \mathrm{C}$ org e Al. Pela análise conjunta dos gráficos dos escores e dos pesos (Gráfico 2), percebe-se que valores altos de $\mathrm{C}$ org e de teor de Al deslocam as amostras com alta topografia (pontos em A) para o lado esquerdo do gráfico dos escores. Por outro lado, valores altos de teor de $\mathrm{Ca}$ e $\mathrm{Mg}$ e de $\mathrm{pH}$ deslocam as amostras para o lado direito do gráfico dos escores, principalmente para o lado superior direito. Observase também que as amostras com os menores valores na Tabela 1 se localizam no centro inferior do gráfico de escores, onde a correlação entre os atributos estudados é menor.

Os níveis de $\mathrm{P}$ e $\mathrm{K}$ mostram-se mais significativos na segunda componente, no entanto essas variáveis não são responsáveis para a classificação das amostras de solo em duas classes: alta topografia e topografias média e baixa. 


\section{CONCLUSÃO}

Os resultados apresentaram níveis de $\mathrm{pH}$ correspondentes aos valores estabelecidos para Latossolos.

A baixa disponibilidade de carbono orgânico indica pouca quantidade de matéria orgânica, uma possível consequência de fenômenos de erosão e arraste de material de serapilheira proveniente da vegetação local.

Os valores de fósforo e potássio apresentaram-se em um nível alarmantemente baixo, estando relacionados aos processos de inundação e lixiviação, constituindo um solo degradado e inviável para o uso na agronomia.

Os solos nas ilhas podem ser classificados como pouco férteis, necessitando de estudos acerca de seu uso e ocupação, bem como uma análise aprofundada dos efeitos de inundação periódica, de forma a estabelecer alternativas para a conservação do ecossistema das ilhas e do lago ao longo das mudanças climáticas e intervenções antrópicas.

\section{REFERÊNCIAS:}

AGÊNCIA DE INFORMAÇÕES EMBRAPA (Empresa Brasileira de Pesquisa Agropecuária).. Abril de $2015 . \quad$ Disponível em: <http://www.agencia.cnptia.embrapa.br/Agencia16/AG01/arvore/AG01_96_1011200 5101956.html>

ARAÚJO, A.R.O.; ROCHA, G.M.. IV Encontro Nacional da Anppas, Brasília-DF, 2008. Disponível em: <http://www.anppas.org.br/encontro4/cd/ARQUIVOS/GT2-604343-20080523212649.pdf>.

BORGES, F.O.; SILVA, S.A.S.; CAMPOS, Y.O.; LEÃO, F.M.; Atributos químicos do solo da mata ciliar do Igarapé Santos, em Tucuruí - PA. Enciclopédia Biosfera, Centro Científico Conhecer, v. 10, n. 19; p. 769-777. Goiânia-GO, 2014.

CAETANO, J.O. VEGINASSI, A.; ASSIS, P.C.R.; CARNEIRO, M.A.C.; PAULINO, H.B.; Indicadores de qualidade de um Latossolo vermelho sob diferentes sistemas de uso e manejo. Global Science and Technology, Rio Verde, v. 6, n. 1, p. 26-39, jan.-abr 2013. Disponível em: <http://dx.doi.org/10.14688/1984-3801>. doi: 10.14688/1984-3801

CARNEIRO, M.A.C.; SOUZA, E.D.; REIS, E.F.; PEREIRA, H.S.; AZEVEDO, W.R. Atributos físicos, químicos e biológicos de solo de cerrado sob diferentes sistemas de uso e manejo. Revista Brasileira de Ciência do Solo, v. 33, p. 147-157, 2009. Disponível em: <http://dx.doi.org/10.1590/S0100-06832009000100016>. doi: $10.1590 /$ S0100-06832009000100016

EMBRAPA - Empresa Brasileira de Pesquisa Agropecuária. Sistemas de Produção, $1^{\mathrm{a}}$ Ed., set. 2009.

EMBRAPA - Empresa Brasileira de Pesquisa Agropecuária. 8ª edição, versão eletrônica, out. 2012.

EMBRAPA - Empresa Brasileira de Pesquisa Agropecuária. Manual de métodos de análise de solos. $2^{\mathrm{a}}$ ed. Rio de Janeiro, RJ: Embrapa Solos. 230p. 2011. 
IBGE - Instituto Brasileiro de Geografia e Estatística; Portal IBGE Cidades, Pará, Tucuruí. Publicado em: agosto de 2014.

JATOBÁ, S.U.S.; Gestão do território e a produção da socionatureza nas ilhas do lago de Tucuruí na Amazônia brasileira. Tese (Doutorado), Centro de Desenvolvimento Sustentável, Universidade de Brasília, DF, 2006.

KARLEN, D.L.; MAUSBACH, M.J.; DORAN, J.W.; CLINE, R.G.; HARRIS,R.F.; SCHUMAN, G.E. Soil quality: a concept, definition and framework for evaluation. Soil Science Society America Journal, v.61, n.1, 1997. Disponível em: <http://dx.doi.org/10.2136/sssaj1997.03615995006100010001x>. doi: 10.2136/sssaj1997.03615995006100010001x

LEITE, M.H.S.; COUTO, E.G.; AMORIM, R.S.S.; COSTA, E.L.; MARASCHIN, L.; Perdas de solo e nutrientes num Latossolo Vermelho-amarelo Ácrico Típico, com diferentes sistemas de preparo e sob chuva natural. Revista Brasileira de Ciência do Solo, v. 33, n. 3, p. 689-699, mar.-jun. 2009. Disponível em: <http://dx.doi.org/10.1590/S0100-06832009000300021>. doi: 10.1590/S010006832009000300021

MATOSO, S.C.G.; SILVA, A.N.; FIORELLI-PEREIRA, E.C.; COLLETA, Q.P.; MAIA, $E$. Frações de carbono e nitrogênio de um Latossolo Vermelho-amarelo Distrófico sob diferentes usos na Amazônia brasileira. Acta Amazônica, v. 42, n.2, p.231-40, 2012. Disponível em: < http://dx.doi.org/10.1590/S0044-59672012000200008>. doi: 10.1590/S0044-59672012000200008

NASCIMENTO, P.C.; LANI, J.L.; MENDONÇA, E.S.; ZOFFOLI, H.J.; PEIXOTO, H.T.; Teores e características da matéria orgânica de solos hidromórficos do Espírito Santo. Revista Brasileira de Ciência do Solo, v. 34, p. 339-48, 2010. Disponível em: <http://dx.doi.org/10.1590/S0100-06832010000200007>. doi: 10.1590/S010006832010000200007

PAULA, R. R.; PEREIRA, M. G.; MACHADO, D.L. Atributos químicos e matéria orgânica em complexos florestais periodicamente inundados na restinga da Marambaia - RJ. Ciência Florestal, Santa Maria, v. 23, n. 4, p. 529-38, out.-dez., 2013. Disponível em: < http://dx.doi.org/10.5902/1980509812337>. doi: $10.5902 / 1980509812337$

PLANO DIRETOR DO MUNICÍPIO DE TUCURUÍ. Meio ambiente: modelo digital de $\quad$ elevação. $2015 . \quad$ Disponível em: <http://www.seidurb.pa.gov.br/pdm/tucurui/elevacao_A3.pdf>.

PLANO DIRETOR DO MUNICÍPIO DE TUCURUÍ. Meio ambiente: pedologia. 2015. Disponível em: < http://www.seidurb.pa.gov.br/pdm/tucurui/pedologia_A3.pdf>.

SEPOF. Estatística municipal de Tucuruí - Pará. Secretaria de Estado de Planejamento, Orçamento e Finanças- Instituto de desenvolvimento econômico, social e ambiental do Pará, 2011. Disponível em: http://iah.iec.pa.gov.br/iah/fulltext/georeferenciamento/tucurui.pdf. Acesso em: 03 de julho de 2014. 
SILVA, A. M.; CANUTO, D. S. DE O.; MORAES, M. L. T. DE; BUZETTI, S.; Avaliação das propriedades químicas em solo de cerrado sob reflorestamento ciliar. Revista Floresta, Curitiba, PR, v. 42, n. 1, p. 49 - 58, jan./mar. 2012. Disponível em: <http://dx.doi.org/10.5380/rf.v42i1.26295>. doi: 10.5380/rf.v42i1.26295

SOUSA, J. B.; PIERANGELI, M. A. P.; SERAFIM, M. E.; SOUZA, C. A. Atributos morfológicos, físicos e químicos de solos e processos erosivos nas margens do Rio Paraguai, Pantanal Superior, Mato Grosso, Brasil. Biologia Geográfica, v. 33, n. 1, p. 109-122, jan.-abr., 2015.2 Disponível em: <http://dx.doi.org/10.4025/bolgeogr.v33i1.22580>. 10.4025/bolgeogr.v33i1.22580

SOUZA, V. F.; GOMES, M.; FERNANDES, L. A.; SAMPAIO, R. A.; NASCIMENTO, C. R. Atributos físicos e químicos do solo e uso das terras das ilhas do Rio São Francisco. Caderno de Ciências Agrárias, v. 7, n. 1, p. 34-45, jan.-abr. 2015. Disponível em: <https://seer.lcc.ufmg.br/index.php/ccaufmg/article/view/1145/921>.

VILLA, M. R.; FERNANDES, L. A.; FAQUIN, V. Formas de potássio em solos de várzea e sua disponibilidade para o feijoeiro. Revista Brasileira de Ciência do Solo, v.28, n. 4, p. 649-58, 2004. Disponível em: < http://dx.doi.org/10.1590/S010006832004000400007> doi: 10.1590/S0100-06832004000400007 